\title{
El cine como herramienta de aprendizaje en el aula. Claves de una experiencia docente multidisciplinar en el ámbito económico*
}

\author{
David Pac Salas \\ Universidad de Zaragoza \\ davidpac@unizar.es \\ Nieves García Casarejos \\ Universidad de Zaragoza \\ ngarcia@unizar.es
}

Resumen: En este trabajo se presenta un proyecto docente que utiliza el cine como recurso facilitador del aprendizaje y de la retención de conocimientos. Este tipo de herramientas didácticas ya se ha utilizado con éxito en multitud de ámbitos: física, enfermería, medicina, etc. Este proyecto, respecto a otros, tiene además dos características diferenciadoras: su carácter multidisciplinar y la participación voluntaria en él de los estudiantes. La experiencia se vinculó a dos áreas de conocimiento, Sociología de la Empresa y Organización de Empresas, de las que fue necesario identificar aspectos comunes y relevantes. Los items seleccionados fueron el papel del empleo en la sociedad y la flexibilidad en el trabajo. Los resultados obtenidos permiten concluir que el visionado de las películas ha permitido a los estudiantes involucrados una mejora de los conocimientos en los items seleccionados.

Palabras clave: Cine, sociología de la empresa, organización de empresas, recursos didácticos, multidisciplinar, aprendizaje.

\footnotetext{
* El presente artículo es resultado del proyecto de innovación docente «Sociología, Historia y Economía a través del cine» correspondiente a la convocatoria PIIDUZ 2008-2 de la Universidad de Zaragoza. Asimismo, los autores agradecen la financiación recibida por el MICINN-FEDER a través del proyecto de investigación ECO 2009-09623, así como la colaboración de los grupos de investigación CREVALOR y ETNOEDU reconocidos por la Diputación General de Aragón.
} 


\begin{abstract}
This paper presents a teaching project that uses movies as a resource facilitator of learning and knowledge retention. These kinds of teaching tool have already been successful in a multitude of fields: physics, nursing, medicine, etc. This project has two features that distinguish it from others: its multidisciplinary character and the voluntary participation of the students. The experience was linked to two areas of knowledge «Sociology of Enterprise» and «Business Organization», so common, relevant features had to be identified. The features selected were the role of employment in society and flexibility at work. The results show that watching movies improved students' knowledge in the items selected.
\end{abstract}

Keywords: Cinema, Sociology of Enterprise, Business Organization, Didactic Resources, Multidisciplinary, Learning. 


\section{Introducción}

Como consecuencia del Espacio Europeo de Educación Superior (en adelante EEES), las enseñanzas universitarias están acelerando el cambio metodológico que habían iniciado años atrás. Las nuevas metodologías ponen de manifiesto que no hay un único modo de transmitir ni de adquirir los conocimientos. De hecho, lo que se pretende es un cambio de modelo docente centrado en los procesos de aprendizaje de los alumnos que facilite el desarrollo de competencias (Rodríguez, 2006; Salinas, 2004), para que se produzca el tan deseado ajuste entre las aptitudes de los titulados y las competencias necesarias para desarrollar el trabajo (Delcourt, 1999).

Para conseguirlo, el EEES se plantea la necesidad de combinar distintas estrategias docentes para la adquisición de competencias generales y específicas. Conviene no olvidar, además, que el EEES ha puesto en el centro del proceso de enseñanza-aprendizaje el trabajo desarrollado por los estudiantes. En este caso, la experiencia llevada a cabo pretende que parte del trabajo de los estudiantes se desarrolle en torno a la utilización del cine.

En la actualidad, uno de los problemas detectados para que los alumnos puedan llegar a ser protagonistas de su propio proceso de enseñanza-aprendizaje es la dificultad para la comprensión de conceptos a nivel abstracto. En este sentido, la utilización de medios audiovisuales (a los que los estudiantes están muy habituados) facilita el tránsito de lo concreto (de las escenas cinematográficas) a los constructos abstractos.

La sociedad del conocimiento ha traído consigo un fuerte desarrollo audiovisual. Los estudiantes están sometidos a permanentes estímulos audiovisuales desde su infancia, de los cuales reciben información constante, por lo que obtienen una parte de sus conocimientos a través de estos medios. El cine forma parte de su vida y de su aprendizaje vital.

La introducción de medios audiovisuales en el sistema educativo resulta imprescindible, por tanto, en un sistema dominado por la letra impresa. Por supuesto, después de la letra impresa, el modo de comunicación con mayor influencia y difusión entre la juventud son las imágenes. Estas se pueden convertir en elementos motivacionales si consiguen despertar el interés del alumno por adquirir nuevos aprendizajes, al tiempo que permiten acelerar el proceso de enseñanzaaprendizaje, ya que trasladar al aula experiencias reales ejerce una influencia positiva en la retención y comprensión de lo aprendido.

Por otro lado, es recurrente que, en economía, un mismo concepto se analice desde diversas ópticas en las distintas asignaturas; así, el empleo, el trabajo, la organización del trabajo, la motivación del trabajador, los incentivos, la subcon- 
tratación y la precariedad son conceptos que se trabajan, entre otros muchos, de forma conjunta dentro de una misma materia, pero de forma aislada entre asignaturas. Por ello, se considera prioritario generar una visión transversal y multidisciplinar de los contenidos fundamentales que comparten las distintas materias dentro de una misma titulación.

A través del cine se consigue impulsar una metodología participativa que permite avanzar en los retos anteriores. Por un lado, encontrar nuevos modos de adquirir conocimientos y, por otro, analizar determinados contenidos de la titulación desde un enfoque más plural, al implicar diferentes disciplinas.

Para poner en valor los dos objetivos anteriores se desarrolló una experiencia piloto que utiliza el cine como herramienta para el aprendizaje de los estudiantes, a partir de una estrategia transversal de enseñanza-aprendizaje desde diferentes asignaturas. En concreto, desde dos disciplinas científicas diferentes (Sociología y Organización), a través de las materias Sociología de la Empresa y Organización y Administración de Empresas, impartidas en primer curso de la diplomatura de Empresariales de la Escuela Universitaria de Estudios Empresariales de Zaragoza (en adelante, EUEEZ).

Otros objetivos específicos que se pretenden alcanzar con esta experiencia incluyen las siguientes competencias genéricas: aumentar el interés de los alumnos por aprender más acerca de los ítems que se proponen en el trabajo; mejorar las habilidades de los alumnos para el trabajo en grupo, para la expresión oral mediante la exposición de ideas y razonamientos y para la expresión escrita mediante la capacidad de síntesis, y fomentar la capacidad crítica de los alumnos.

\section{El cine como herramienta de aprendizaje en el aula}

Existen no pocas reflexiones sobre el uso de materiales audiovisuales en la docencia universitaria. ${ }^{1}$ A ello ha contribuido que, en los últimos años, se hayan desarrollado proyectos de innovación docente que, con el objetivo de facilitar la adaptación al Espacio Europeo de Educación Superior, incorporan la utilización de estos materiales. Si bien, en algunas ocasiones, esta utilización se lleva a cabo

\footnotetext{
1 Sin pretensión de exhaustividad, en el área de medicina podemos encontrar experiencias y materiales específicos sobre el uso del cine con fines educativos, discursivos, de mentalización y divulgativos. Véase, para ello, la Revista Medicina y Cine - Journal of Medicine and Movies_, publicada por la Universidad de Salamanca. También, dentro de la enseñanza universitaria, es posible encontrar ejemplos en los estudios de enfermería (Icart-Isern et alii, 2008) y en física (Quirantes, 2011). Otro material interesante es el coordinado por López Ciriano (2006), en el que realiza una recopilación y selección de escenas sobre cuestiones laborales, editado en un formato DVD. Otros recursos disponibles en la red son < http://www.econoaula.com/> y < cineyeconomia. blogspot.com.es $>$, entre otros.
} 
para fomentar la participación en la discusión de temas controvertidos más que como un medio de transmisión de conocimientos y valores.

Por supuesto, existen ejemplos de utilización de este tipo de materiales tanto en la enseñanza de la Sociología como en materias propias del ámbito de la Economía de la Empresa. Así, los profesores Fernández y Olmedo (2003) presentaron una experiencia sobre el apoyo audiovisual para la comprensión de la dimensión social de la enseñanza en la asignatura Sociología de la Educación.

El recurso de materiales audiovisuales puede ser un camino eficaz para presentar de manera comprensible la materia y conseguir que atraiga y despierte el interés de los alumnos hacia ella y les resulte relevante (como ahora se dice). Esto es particularmente importante en los primeros momentos del curso donde la calidad de las primeras impresiones, positivas o negativas, puede ser determinante para la comunicación a lo largo del curso. Además la dificultad es mayor porque en las primeras clases se suele plantear ideas muy globales, generales, más abstractas y difíciles de captar, que constituirán las bases sobre las que iremos construyendo a lo largo del curso (Fernández y Olmedo, 2003: 1).

Dentro de las materias de Economía de Empresa, las profesoras Pérez y López (2007) utilizan el cine dentro de la metodología docente para la enseñanza de los recursos humanos.

A través del cine el alumno/espectador puede recibir información que le ayude a ordenar cognitivamente sus conocimientos, dándole la oportunidad de participar en muchas situaciones diferentes que, de no ser por este medio, no hubiera tenido a su alcance. Los expertos admiten que las películas no son capaces de mostrar la complejidad del mundo financiero y de las transacciones comerciales, pero sí son efectivas cuando tienen que ver con los aspectos humanos de los negocios. El cine propone al espectador una historia, pero no la narra buscando un receptor pasivo, sino que facilita la identificación del espectador con los diferentes matices del discurso. A través de la identificación de los personajes, la trama, la acción, el espectador pasa de ser mero testigo de la narración a convertirse en actor esta. De esta forma el aprendizaje se produce activamente, a través de la experiencia (Pérez y López, 2007:66).

Otro aspecto de interés que se viene analizando es el impacto de lo visual en la memoria. Así, el pedagogo estadounidense Edgar Dale (1932) estableció una jerarquización del aprendizaje en forma de cono. Se trataba de analizar la profundidad del aprendizaje según las actividades docentes realizadas. Según esta jerarquización, la actividad visual (el cine, entre ellas) es más potente que otras para la memorización de información y respecto a la profundidad en el conocimiento adquirido. Esto cobra más sentido cuando se observa, tal y como se ha 
puesto de manifiesto anteriormente, la dificultad que tienen los alumnos para la comprensión de conceptos a nivel abstracto. Por todo ello, la utilización del cine puede mejorar el método docente.

En el modelo de intervención que se desarrolla, los propios estudiantes son los protagonistas. El esquema que se presenta a continuación muestra, grosso modo, los pasos que seguir para cada uno de los visionados:

Figura 1. Modelo de intervención docente

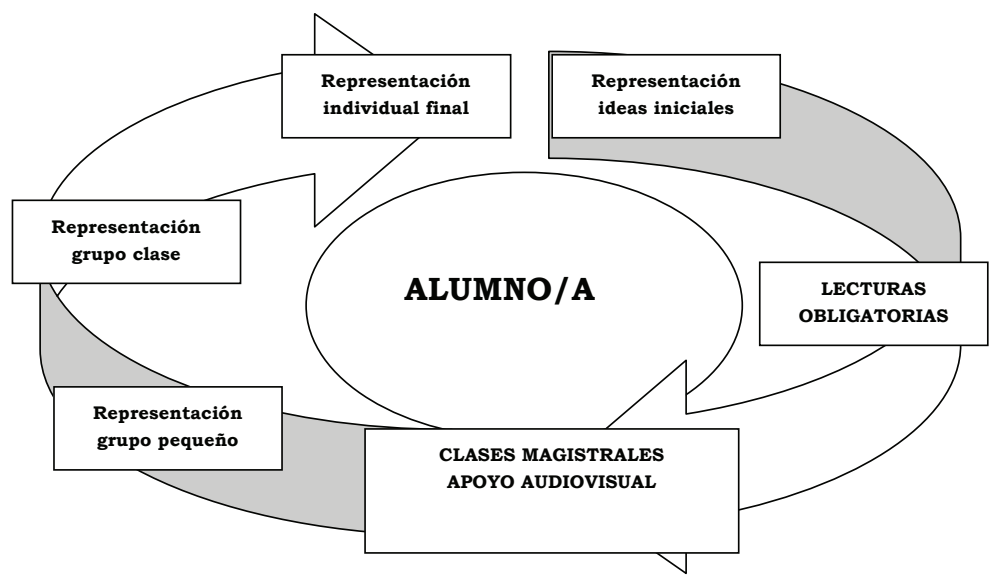

En nuestro caso la intervención con apoyo audiovisual ha consistido en dos visionados. Las películas seleccionadas son La cuadrilla (2001), de Ken Loach, y Los lunes al sol (2002), de Fernando León de Aranoa.

\section{Desarrollo del proyecto}

El proyecto se articuló como una actividad voluntaria vinculada a dos asignaturas, Organización y Administración I y Sociología de la Empresa, troncal la primera y optativa la segunda. Las asignaturas pertenecen a dos áreas de conocimiento distintas, dado el carácter transversal que se pretendía imprimir al proyecto. El proyecto se realizó durante el mes de mayo de 2009, y en él participaron 32 alumnos de la asignatura Organización y Administración de Empresas I, al ser la única con docencia en dicho cuatrimestre.

El contacto con los posibles alumnos interesados en el proyecto se llevó a cabo mediante una carta de presentación del proyecto que se entregó a los cuatro grupos de docencia de la asignatura Organización y Administración I, y en la que 
se explicaban los objetivos del proyecto, las actividades que debían realizar los alumnos, su temporalización y el método de evaluación.

Los alumnos participantes en esta experiencia eran estudiantes de primer curso de la diplomatura y, por tanto, todavía no habían consolidado determinados constructos, en concreto, aquellos que se planteaba analizar en este trabajo. Por ello, se esperó a mayo para que hubieran visto en las aulas los conceptos referidos a los temas de estudio asociados a esta experiencia.

Respecto a las tareas que ha conllevado la realización del proyecto, habría que distinguir entre las que realizaron los dos profesores vinculados a la experiencia y las que tuvieron que realizar los alumnos.

\subsection{Actividades realizadas por los profesores}

Las actividades realizadas por los profesores fueron:

+ Diseño del proyecto

- Difusión del proyecto entre los posibles alumnos interesados

+ Selección del alumnado

- Elección de ítems a valorar en cada una de las películas

- Contacto con los alumnos por medio del correo electrónico o del teléfono.

- Diseño de cuestionarios previsionado y posvisionado a través de la herramienta Google Docs

+ Evaluación de los resultados

\subsection{Actividades realizadas por los estudiantes}

Las actividades principales se concentraron en dos sesiones:

Primera reunión

Fecha: martes 5 de mayo de 2009

Hora: de $15: 30$ a $19: 30$

Lugarः salón de actos

Programa de actividades

- Presentación del proyecto

- Comentarios sobre el autoinforme previo sobre conocimientosः trabajo y organización del trabajo

- Visionado de la película Los lunes al sol (2002)

+ Práctica en grupos de 2-3 personas

- Puesta en común en el grupo 


\section{Segunda reunión}

Fecha: martes 19 de mayo de 2009

Hora: de $15: 30$ a 19:30

Lugar: salón de actos

Programa de actividades

- Visionado de la película La cuadrilla (2001)

- Práctica en grupos de 2-3 personas

- Puesta en común en el grupo

- Autoinforme del seguimiento de la actividad

- Cuestionario de evaluación de toda la actividad

De los 32 alumnos que decidieron participar en la experiencia (todos ellos de la asignatura Organización y Administración I), ocho (el 25\%) lo fueron anteriormente de Sociología de la Empresa, lo que permitió que existiera un grupo de control, puesto que estos alumnos ya habían trabajado en la asignatura, durante el primer cuatrimestre, buena parte de los ítems que se presentan en esta experiencia y, por tanto, se esperaba que tuvieran un mayor conocimiento inicial y que el incremento de dominio del concepto fuera menor.

Previamente al visionado de cada una de las películas, los profesores entregaban a los alumnos una ficha con las preguntas que deberían responder posteriormente. A continuación, los profesores presentaban la película que se iba a proyectar con objeto de dar sentido a la actividad y relacionarla con los temas que tratar. Las proyecciones se realizaron en el salón de actos de la EUEEZ. Tras finalizar la proyección, se entraba en la reflexión y debate alrededor de cada uno de los ítems. Los profesores, durante el debate, adoptaban el papel de orientador, moderador y mediador.

En cuanto a los aspectos técnicos de las películas que se visionaron dentro de la experiencia que se recoge en este trabajo, se podría decir que, aunque ambas mantienen importantes coincidencias respecto al enfoque con el que abordan las cuestiones que plantean, también se aprecian sutiles diferencias. La cuadrilla es una coproducción británica, española y alemana que pertenece a la categoría de cine de denuncia, dirigida por uno de los autores más prolíficos en la materia, Ken Loach. El film trata el tema de las privatizaciones de los ferrocarriles llevadas a cabo en Inglaterra, a mediados de la década de los 90. Por su parte, Los lunes al sol es una película cien por cien española, del director Fernando León de Aranoa, de elevado contenido social y con un humor en ocasiones mordaz, que plasma de una forma muy realista el difícil contexto humano del parado. 
Ficha técnica de la películaः Los lunes al sol

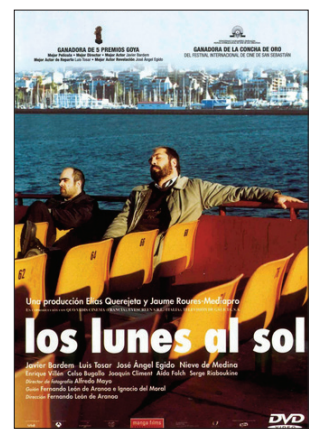

Dirección: Fernando León de Aranoa.

Año: 2002.

País: España.

Duración: $113 \mathrm{~min}$.

Interpretación: Javier Bardem (Santa), Luis Tosar (José), José Ángel Egido (Lino), Nieve de Medina (Ana), Enrique Villén (Reina), Celso Bugallo (Amador), Joaquín Climent (Rico), Aida Folch (Nata), Serge Riaboukine (Serguei), Laura Domínguez (Ángela), Pepo Oliva (Samuel), Fernando Tejero (Lázaro).

Guión: Fernando León de Aranoa e Ignacio del Moral.

Sinopsis: Una ciudad costera del norte, dividida por una ría de aguas verdes y oleaginosas. Muchos hombres y mujeres dejaron atrás el campo o el mar para ir a trabajar a las fábricas, a las refinerías, al astillero. Pero después llegó la reconversión industrial. En el bar de Rico se reúne un grupo de amigos. Conversan en las horas muertas, se juegan sus esperanzas en la máquina tragaperras... en el bar se mezclan los recuerdos y los proyectos, se comparten las frustraciones y las esperanzas. Como un fantasma, el cierre del astillero planea sobre ellos. En su calendario todos los días son festivos, pero en todos hay motivo para la desesperación.

Ficha técnica de la película: La cuadrilla

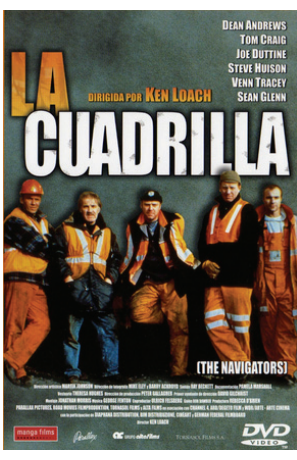

Guión: Rob Dawber.

Sinopsis: Un grupo de trabajadores encargados del mantenimiento de las infraestructuras ve cómo su situación laboral queda amenazada por criterios meramente productivos. Son invitados a firmar «despidos voluntarios», pierden su derecho a vacaciones, oyen hablar de una flexibilidad laboral que encubre en realidad la precariedad en el trabajo, y ven cómo se reducen los gastos - lo que se traduce en menos puestos de trabajo - con el consiguiente aumento del riesgo. 


\section{Resultados}

La evaluación de la experiencia se ha llevado a cabo mediante dos estrategias: por un lado, la propia evaluación de la experiencia realizada por los estudiantes; por otro, la evaluación realizada por los profesores sobre los resultados del aprendizaje alcanzados por los estudiantes. En este apartado se hará referencia a estos dos aspectos.

\subsection{La evaluación de los estudiantes}

Para evaluar el grado de satisfacción de los estudiantes con la experiencia docente se elaboraron dos cuestionarios, uno al principio de las sesiones y otro al finalizar el proyecto.

En la sesión inicial los alumnos elaboraron un autoinforme sobre diversos aspectos objetivables - cuestiones curriculares, asistencia al cine, participación en otras experiencias y variables sociodemográficas-, y aspectos subjetivos - como expectativas iniciales y motivaciones para participar en la experiencia docente.

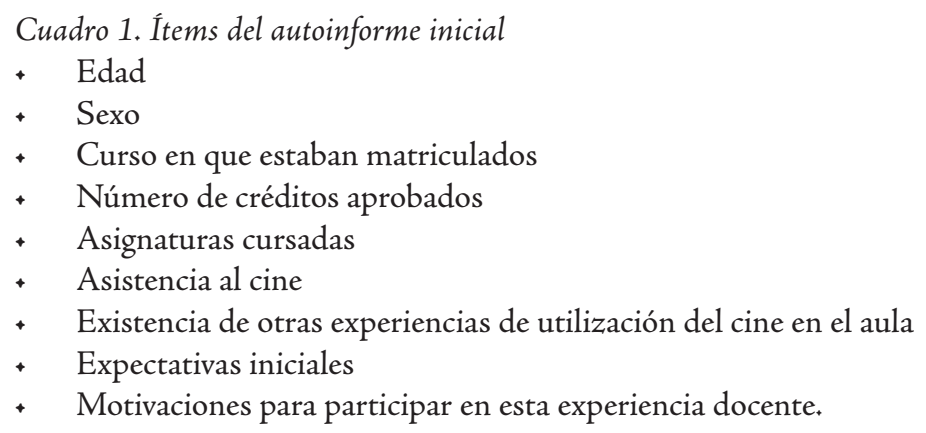

En la sesión final el cuestionario incluía cuestiones subjetivas referidas al grado de cumplimiento de las expectativas iniciales y de satisfacción con los objetivos alcanzados, tanto de carácter metodológico como de aprendizaje. De forma más detallada, en la última sesión se pretendía conocer mediante el autoinforme el grado de cumplimiento de las expectativas iniciales, la valoración del proceso y desarrollo del proyecto y el grado de satisfacción con este. Por último, se indagaba sobre los conceptos aprendidos, los aspectos positivos y negativos de la experiencia y las propuestas de mejora para futuras ediciones.

En general, la valoración de los estudiantes de la experiencia docente ha sido muy positiva. Así, ante la pregunta: «¿Recomendarías a otros compañeros la par- 
ticipación en esta experiencia en futuras ocasiones?», todos ellos contestan afirmativamente, es decir, recomendarían la actividad a otro compañero/a.

En la construcción de los cuestionarios se utilizaron dos tipos de preguntas: cerradas (de escala) y abiertas. Para las preguntas cerradas se utilizó una escala Likert con 10 niveles de respuesta. En este tipo de preguntas la valoración media que dieron los alumnos en los distintos ítems fue de 7 sobre 10. Así, por ejemplo, los estudiantes manifiestan un alto grado de cumplimiento de las expectativas iniciales, como demuestra el hecho de que a la pregunta «Mis expectativas iniciales con este proyecto de innovación se han visto...», la respuesta promedio fue 7,1 . Además, consideran que el desarrollo del proyecto de innovación ha sido adecuado respecto a los objetivos diseñados. La valoración del desarrollo (diferencias entre el diseño inicial y la propuesta final) del proyecto de innovación tuvo una valoración media de 7,2.

\section{Cuadro 2. Items del autoinforme final}

- Mis expectativas iniciales con este proyecto de innovación se han visto...

- El desarrollo del proyecto de innovación ha sido....

+ En el proceso del proyecto de innovación docente...

+ ¿Qué conceptos has aprendido a través de la experiencia?

+ ¿Qué grado de satisfacción tienes con el proceso de innovación desarrollado?

- Señala las aspectos POSITIVOS del proyecto para el aprendizaje de conceptos económicos y sociológicos

- Señala las aspectos NEGATIVOS del proyecto para el aprendizaje de conceptos económicos y sociológicos

- ¿Recomendarías a otros compañeros la participación en esta experiencia en futuras ocasiones?

- Señala las aspectos que consideras necesarios modificar para el aprendizaje a través del cine

Dentro de las preguntas abiertas, los alumnos consideran que los aspectos positivos en el aprendizaje a través de películas son: la facilidad para aprender y la amenidad, puesto que «te enseña a ver las cosas estudiadas en un entorno real de la sociedad»y lo definen como «una forma diferente y rápida para la transmisión de los conocimientos».

\subsection{Los resultados alcanzados en el aprendizaje}

Para llevar a cabo la evaluación se implementaron varios indicadores. Los estudiantes rellenaban un cuestionario, diferente para cada película, a través de la herramienta Google Docs. Cada uno de los cuestionarios se sometía a la consi- 
deración de los alumnos dos veces, una antes del visionado de la película y otra inmediatamente después. El objetivo era observar, sin interferencias, los cambios que se habían producido en sus conocimientos sobre los aspectos e ítems considerados en cada película.

En cada cuestionario, formado por preguntas abiertas, se indagaba sobre los conceptos y elementos sociológicos y económicos que se podían observar en cada una de las dos películas. En los siguientes cuadros ( 3 y 4 ) se recogen las preguntas formuladas para cada una de las películas y que conforman los ítems analizados en cada una de ellas.

Cuadro 3. Ítems Los lunes al sol

$¿$ Qué dota de identidad a los individuos?

¿Qué papel cumple el empleo en la sociedad?

¿Qué satisfacciones obtienen las personas a través del trabajo?

¿Qué diferencias observas entre el trabajo que realizan los hombres y las mujeres?

$¿ Q u e ́$ factores tenemos que tener en cuenta ante una reestructuración/cierre de una empresa?

Ante el desempleo, ¿qué diferentes actitudes tienen las personas?

¿Qué repercusiones tiene el desempleo?

Cuadro 4. Ítems La cuadrilla

¿Qué cambios económicos, políticos y sociales se han producido en el entorno empresarial en los últimos treinta años?

Enumera las repercusiones que los cambios anteriormente señalados han supuesto para las empresas

¿Qué consecuencias produce la privatización de empresas públicas?

$¿$ Qué entiendes por flexibilidad en el entorno laboral?

$¿$ ¿ué implicaciones tiene la flexibilidad?

$¿$ ¿ué función cumplen los sindicatos?

Los resultados para ambas películas (entre el cuestionario pre-y posvisionado) muestran cómo, en general, se produce un incremento en los conocimientos en todos los ítems seleccionados. El grado del incremento en los conocimientos ha sufrido variaciones por dos motivos: 1) debido a la complejidad y abstracción de los conceptos que analizar, y 2) según los conocimientos previos de los alumnos. ${ }^{2}$

2 Se constató que el grupo de alumnos que había cursado ya la optativa «Sociología de la Empresa» poseía mayores conocimientos previos que sus compañeros/as en conceptos e ítems sociológicos, lo cual se vería reflejado en sus respuestas previsionado. 
Para ilustrar estas afirmaciones se presentan algunos ejemplos. En el visionado de la película Los lunes al sol, la primera cuestión que se planteaba a los alumnos era «¿Qué dota de identidad a los individuos?». En las respuestas previsionado de la película, la mayoría de los alumnos asimilaban la identidad con la personalidad de los individuos, lo que pone de manifiesto carencias en cuanto a sus conocimientos previos. El visionado de la película les permite incorporar elementos sociológicos, por ejemplo: la posición social, el empleo, las relaciones sociales. Esto puede apreciarse en las siguientes respuestas:

- Respuesta previsionado: «La posición social, una buena formación, y por lo tanto un trabajo estable, para poder llevar una vida digna».

- Respuesta posvisionado: «La situación en la que se encuentran en cada momento. En la película los individuos se sentían identificados con su trabajo y su familia (quienes la tienen). Al perder el trabajo sienten como si perdieran parte de su identidad. Los actos que realizan día a día y su forma de pensar cambian y se adaptan a su situación laboral. Por ejemplo, cuando los personajes tenían trabajo se servían de este para establecer su rutina y sus relaciones personales (con compañeros). Al perderlo les cambia el carácter y la forma de ver las cosas. Asimismo, el trabajo y los problemas que de este se derivan sirven para estrechar las relaciones entre compañeros y en ocasiones también para distanciarlas».

La siguiente cuestión que se planteaba a los estudiantes era «¿Qué papel cumple el empleo en la sociedad?». En su representación inicial hacen hincapié, sobre todo, en el valor instrumental del empleo: la obtención de rentas económicas a través del trabajo (salario); es decir, hay una identificación entre empleo e ingresos. Sin embargo, en su representación posterior incorporan otros elementos más sociológicos: estatus, hombre como ser social, etc.

- Respuesta previsionado: «Las personas necesitan el empleo para poder vivir en la sociedad, ya que la contrapartida de estar empleado es un salario con el que poder vivir».

- Respuesta posvisionado: «Es un papel muy importante, ya que sin trabajo, no hay dinero, sin dinero no puedes pagar gastos, comida... El empleo es también una forma de relacionarse socialmente y tener un «estatus» en la sociedad (en tu círculo social). Teniendo empleo tienes seguridad, económica sobre todo, pero también personal, te sientes realizado profesionalmente y aceptado en un grupo, ya que el hombre es un ser social por naturaleza».

Por último, hay que destacar el valor de las imágenes como refuerzo positivo para comprender las distintas actitudes ante el desempleo. Para la pregunta, 
«Ante el desempleo, ¿qué diferentes actitudes tienen las personas?», los alumnos muestran un mayor caudal de conocimiento.

- Respuesta previsionado: «Depresión, intención de formarse, desesperación».

- Respuesta posvisionado: «Se puede afrontar de tres maneras: en primer lugar se puede afrontar con optimismo e intentando encontrar un nuevo trabajo. Es el caso de Lino en la película. Se puede recibir formación e intentar obtener un nuevo empleo en el menor tiempo posible para subsanar las consecuencias que tiene la pérdida de este. Por último, se puede afrontar con resignación y derrotismo. Conformándose con la situación que se produce tras la pérdida del empleo. Esta postura puede acabar en depresión (caso de suicidio de la película). Otra posible actitud puede ser la evasión (caso de Santa)».

De la película La cuadrilla, se procede a destacar otros tres ejemplos que permitan mostrar la ampliación y consolidación de conocimientos por parte de los estudiantes. En primer lugar, en referencia a la pregunta «QQue consecuencias produce la privatización de empresas públicas?», el cine proporciona nuevas dimensiones sobre el sentido y las consecuencias de las privatizaciones, e incluso permite asentar o modificar presunciones previas sobre estas, sustentadas, en ocasiones, por lugares comunes o estereotipos.

- Respuesta previsionado: «La privatización fundamentalmente es desmontar el Estado y dejar a los particulares brindar libremente servicios que anteriormente habían sido monopolizados por el Estado».

- Respuesta posvisionado: «Con la privatización las empresas recortan gastos y desean que se produzcan cambios radicales en la forma de gestionar la empresa. Exigen, además, la reducción del número de accidentes laborales y que no exista mano de obra ociosa. Los materiales ya no son adquiridos por la empresa sino que corren por cuenta de los trabajadores, las condiciones de trabajo empeoran, aumentan los despidos y los contratos basura. Reducen los salarios y a los que no se adapten a sus cambios se les despide».

Otro ámbito donde se muestran avances relevantes es el del conocimiento de los sindicatos («¿Qué función cumplen los sindicatos?»). En general, en las encuestas que pretenden medir la confianza de los ciudadanos en las instituciones, estos muestran poca confianza en ellas. La percepción inicial de los estudiantes actuales no dista mucho de esa visión general. Sin embargo, a través de los acontecimientos que se muestran en la película, mejora la percepción de los alumnos sobre el papel de representación de los trabajadores que ostentan los sindicatos. 
Respuesta previsionado: «Defender los derechos de los trabajadores».

Respuesta posvisionado: «Los sindicatos se encargan de representar a los trabajadores y sus derechos, luchan por alcanzar un alto nivel de bienestar en la empresa, por conseguir convenios para mejorar las horas extras, vacaciones, jornada laboral, etc. Aunque en muchos casos los sindicatos están cargados de ideas políticas que dificultan el desarrollo de la empresa, ya que nunca están satisfechos».

El último aspecto que permite ejemplificar la mejora de conocimientos que se produce tras el visionado de la película La cuadrilla son las repercusiones que sobre las empresas tienen los cambios económicos. Entre estos cambios se encuentra la privatización de los ferrocarriles británicos, al incidir en la propia organización y en las condiciones de trabajo de los empleados.

Respuesta previsionado: «Los avances tecnológicos adquieren un papel más importante en nuestras empresas facilitando la producción y reduciendo el factor humano, al mecanizarse los procesos productivos».

Rrespuesta posvisionado: «La privatización del ferrocarril ofrece condiciones bastante desfavorables a los trabajadores, para invitarles a abandonar el puesto de trabajo (cobrando una indemnización bastante insignificante) o continuar en la empresa privatizada pero bajo las nuevas condiciones establecidas, que no siempre son apoyadas por los trabajadores. A la seguridad laboral, a pesar de ser un aspecto clave en el trabajo, no se le da la importancia que debería. Todos estos aspectos influyen desde el punto de vista económico».

\section{Conclusiones}

La experiencia que se presenta en este texto muestra el valor añadido de la utilización del cine en el aula universitaria. En el modelo de aprendizaje que conlleva la adaptación al Espacio Europeo de Educación Superior se recomienda utilizar distintas estrategias y herramientas de enseñanza-aprendizaje: clases magistrales, trabajo en grupos pequeños, trabajo individual, etc. En nuestro caso, la evaluación del efecto del visionado de las películas en la mejora de los conocimientos en los ámbitos de la sociología y la organización de empresas, tanto por parte de los alumnos como de los profesores, ha resultado muy positiva.

En concreto, a partir de la valoración positiva que los estudiantes conceden a esta experiencia, cabría destacar la conveniencia de abordar los conceptos de forma multidisciplinar en lugar de abordarlos de forma aislada, ya que el aprendizaje transversal de la empresa permite transformar un modelo basado en conocimientos aislados (que deriva en el asignaturismo) en un aprendizaje integral (pensar y comprender la globalidad de la empresa). Asimismo, pone de manifiesto la im- 
portancia de la motivación en el proceso de aprendizaje, así como la importancia de incorporar medios audiovisuales, dado que permiten acelerar el proceso de enseñanza-aprendizaje.

Como propuesta de futuro, convendría seguir avanzando en la integración de estas experiencias y recursos didácticos en el modelo docente establecido en las distintas materias del ámbito económico.

\section{Bibliografía}

Cine y Economía IES Beb Gabirol. Disponible en: <cineyeconomia.blogspot.com. es $>$.

Dale, E. (1932). «Methods for Analyzing the Content of Motion Pictures». Journal of Educational Sociology, 6: 244-250.

Delcourt, J. (1999). «Nuevas presiones a favor de la formación en la empresa». Formación Profesional, 17, 3-14.

Fernández Palomares, F. y Olmedo Reinoso, A. (2003). «Barbiana y las «escuelas japonesas»: apoyos audiovisuales para ayudar a comprender la dimensión social de la enseñanza», X Conferencia de Sociología de la Educación, Valencia, 18-20 septiembre.

ICART-Isern, M.T. y ACOPI (Grupo de Innovación Docente) (2008). «Metodología de la investigación y cine comercial: claves de una experiencia docente». EDUC MED, 11 (1): 13-18.

López Ciriano, M. A. (2006). Cine, orientación profesional y empleo. DVD. Gobierno de Aragón.

Quirantes Sierra, A. (2011). «Física de película: una herramienta docente para la enseñanza de física universitaria usando fragmentos de películas». Revista Eureka sobre Enseñanza y Divulgación de las Ciencias, 8 (3): 334-340, Universidad de Cádiz. Disponible en: <http //rodin.uca.es $\$ 8081 /$ xmlui/ handle/10498/14397>.

Pérez López, S. y López Mielgo, N. (2007). «Metodología docente para la enseñanza de los recursos humanos: el uso del cine». Aula Abierta, 35, 1 y 2: 63-76. Disponible en: <http://www.uniovi.net/ICE/publicaciones/Aula_ Abierta/numeros_anteriores/i8/063_pdfsam_AulaAbierta_35_2007.pdf $>$.

Rodríguez, R. (2006). «Diseño de entornos para el desarrollo de la autonomía en el aprendizaje». Aula Abierta, 87, 89-104. Disponible en: dialnet.unirioja. es/servlet/fichero_articulo?codigo $=2583893$.

Salinas, J. (2004). «Innovación docente y uso de las TIC en la enseñanza universitaria». Revista Universidad y Sociedad del Conocimiento, 1 (1), noviembre. 
Web educativa e informativa de economía y sociedad. <http://www.econoaula. $\mathrm{com} />$.

\section{Películas citadas en el presente trabajo:}

Los lunes al sol (C) Manga Films S.L., 2002

La cuadrilla (C) Manga Films S.L., 2001 